\title{
RBF Neural Network-Based Prediction and Inverse Calculation of Air Pollutant Emission Concentration
}

\author{
Zheng Xipeng ${ }^{1}$, Yang Shunsheng ${ }^{1}$, Xiang Wenchuan ${ }^{1}$, Chen $\mathrm{Yu}^{2}{ }^{2}$, \\ ${ }^{1}$ School of Civil Engineering, Southwest Jiaotong University, Chengdu, China \\ ${ }^{2}$ Faculty of Geosciences and Environmental Engineering, Southwest Jiaotong University, Chengdu, China
}

\section{Email address:}

273620202@qq.com (Chen Yu)

${ }^{*}$ Corresponding author

\section{To cite this article:}

Zheng Xipeng, Yang Shunsheng, Xiang Wenchuan, Chen Yu. RBF Neural Network-Based Prediction and Inverse Calculation of Air Pollutant Emission Concentration. American Journal of Biological and Environmental Statistics. Vol. 4, No. 2, 2018, pp. 66-73. doi: 10.11648/j.ajbes.20180402.13

Received: June 26, 2018; Accepted: July 16, 2018; Published: August 9, 2018

\begin{abstract}
The arrangement of the sensors in the air pollutant distribution space was designed by segmented array. A data prediction model for RBF neural network was created. Other air pollution data at the unknown positions were predicted by the data measured by the arranged sensors in order to reduce the sensor arrangement cost. According to the measured values and the predicted data, Gaussian plume diffusion model for air pollution was created, and the quadratic optimization model and inversion method for inverse calculation of single pollution source and multi pollution source were built. Single pollution source and double pollution source was inversely optimized by three different intelligent optimized algorithms in experimental simulation in order to obtain the accurate information on pollution sources. The validity of this method was verified so as to provide a reference for subsequent research.
\end{abstract}

Keywords: Air Pollution, Sensor, Gaussian Plume Diffusion Model, Intelligent Optimized Algorithm

\section{Introduction}

In recent years, the increasing vehicles and factories and mines consume a lot of fossil energy, resulting in increasing air pollutant emissions, and more and more serious air pollution [1]. In fact, pollutant diffusion in air is very complicated and involved in several space dimensions [2]. In addition, pollutant type, meteorological condition, and geographic location all influence the pollutant distribution. As two main factors of influencing the pollutant diffusion, pollution source position and intensity have been experimentally and simulatively studied by different researchers.

The neural network model has been used for inverse calculation of air pollutants more often because of its advantages, including low resource consumption, more acquired data, etc., over the measured pollutant data. Although the air pollution value is predicted based on basic equation of air diffusion, the accuracy of numerical solution is influenced by different aspects, resulting in accuracy difference, including (1) initial errors determined by initial field, such as instrument error during measurement, and adverse representative influence of instrument mounting position, etc.; (2) numerical pattern randomicity: the difference between dynamic pattern or chemical mechanism pattern and real air always exists to some extent, certainly resulting in the deviation of the predicted result from real air; (3) intrinsic randomicity of air motion process: the average wind speed and wind direction measured at different points in the meso scale flat area naturally vary randomly because of turbulent flow; and (4) uncertainty of pollution source intensity and parameters: the random change of pollution source intensity certainly greatly increases the difficulty in air pollution prediction and discreteness of prediction results. Thus, the current studies on neural network model-based accurate calculation of pollutants still are facing many difficulties [3]. Different researchers' inverse calculation of pollutant source concentrations and positions by the pollutant concentration data and meteorological condition measured by the sensors in the space showed that inverse calculation could be achieved on the premise of enough pollutant concentration distribution data [4-6]. The method of solving the problems about inverse calculation of pollution sources mainly include: (1) Gaussian plume diffusion model-based inverse calculation of pollution sources by intelligent optimized algorithm, which always applies to pollutant 
diffusion on flat landform and within several miles [7]: Annunzio [8] and Chen Junming et al. [9] used the genetic optimized algorithm to calculate the position and source intensity a single pollution source; Zhang Jiufeng et al. [10] used the particle swarm optimization algorithm for inverse calculation of pollution source, and studied the effect of each parameter on the optimization efficiency in order to reduce the inverse calculation cost; (2) Use of Kalman filtering [11] and Lagrangian Particle [12] for calculation of key data of the air pollution diffusion model in order to reconstruct the model, with some results: Shen Xiaoyan et al. [13] established the equation of gas concentration vs. time as a state equation, and used Kalman filtering for improvement to effectively eliminate noise interference during monitoring; (3) Bayesian Probability-based reconstruction of the air pollution diffusion model [7, 14, 15]: Johannesson et al. [16] used Markov chain and Monte Carlo method for reconstruction of the air pollution model, with some results; Senoca et al. [17] created a Bayes' theorem-based data driven approach to calculate the parameters of Gauss plume diffusion model for turbulent flow, but Feng Fan et al. [18] believed that such statistics-based method was very sensitive to observation error, posterior estimation error, etc.; and (4) use of the parallel computation method for air pollution in 3-D space to longitudinally divide the space into several layers, each of which is divided into several grids according to the same resolution in order to perform inverse calculation of air pollutant emission at each layer [19, 20]. Such method can effectively solve such problems about inverse calculation of air pollution as low efficiency, long time, etc.

However, few studies focus on inverse calculation of pollutants by neural network, and most of them aim at a single pollution source and has insufficient analysis and comparison of different intelligent optimized algorithms. To solve the problem, the available air pollution concentration data were used to predict the pollutant concentration at the unknown positions; the built mathematic model for inverse calculation of multi pollution source intensity was used to build a constrained optimized algorithm; the errors between available downwind air pollutant concentration and predicted air pollutant concentration and measured concentrations could be regarded as the value of target function; intelligent algorithm was used to find the optimum solution in order to enhance the calculation efficiency and reduce the inverse calculation cost in this paper.

\section{Research Method}

\subsection{RBF Neural Network-Based Data Prediction Model}

RBF neural network, which was initiated in 1988 [21], has good generalization ability and a simple network structure, and can approach any nonlinear function at any accuracy $[22,23]$. RBF neural network has an input layer, hidden layer and output layer. The neuron activation function of hidden layer is a radial basis function, $h(t)$, and its structural formula is shown in Formula (1):

$$
h(t)=\exp \left(-\frac{\|\delta x(t)\|^{2}}{2 \cdot b^{2}}\right)
$$

Where

$\delta x(t)$ : Euclidean distance between input parameter vector and central vector;

$b:$ Width of Gaussian function.

The activation function from the hidden layer to output layer is calculated from the following linear weighting function:

$$
y(t)=\sum \omega \cdot h(t)
$$

Where

$\omega$ : Weight of the output layer.

The Gaussian plume diffusion model-based experimental data were used to create a neural network model. The first 200 groups of data were used for a drill, and the rest 200 groups of data were used for comparative simulation of the predicted results. The value of concentration was too low, and even about $10^{-5}$ during operation. The relative error was very big at the absolute error of $10^{-5}$, so the concentration data were normalized first. The comparative simulation results from RBF neural network-based data prediction and BP neural network-based data prediction were shown in Figure 1 3.

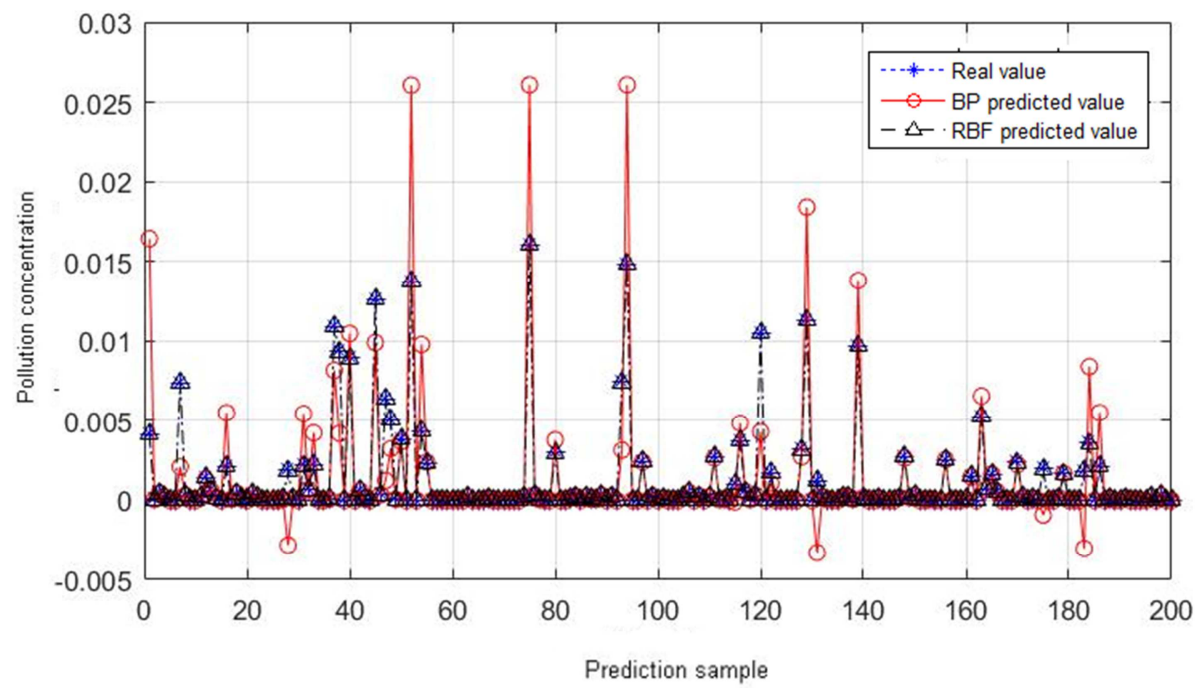

Figure 1. Comparison of BP $\mid R B F$-based Pollutant Concentration Prediction Results And Actual Value. 

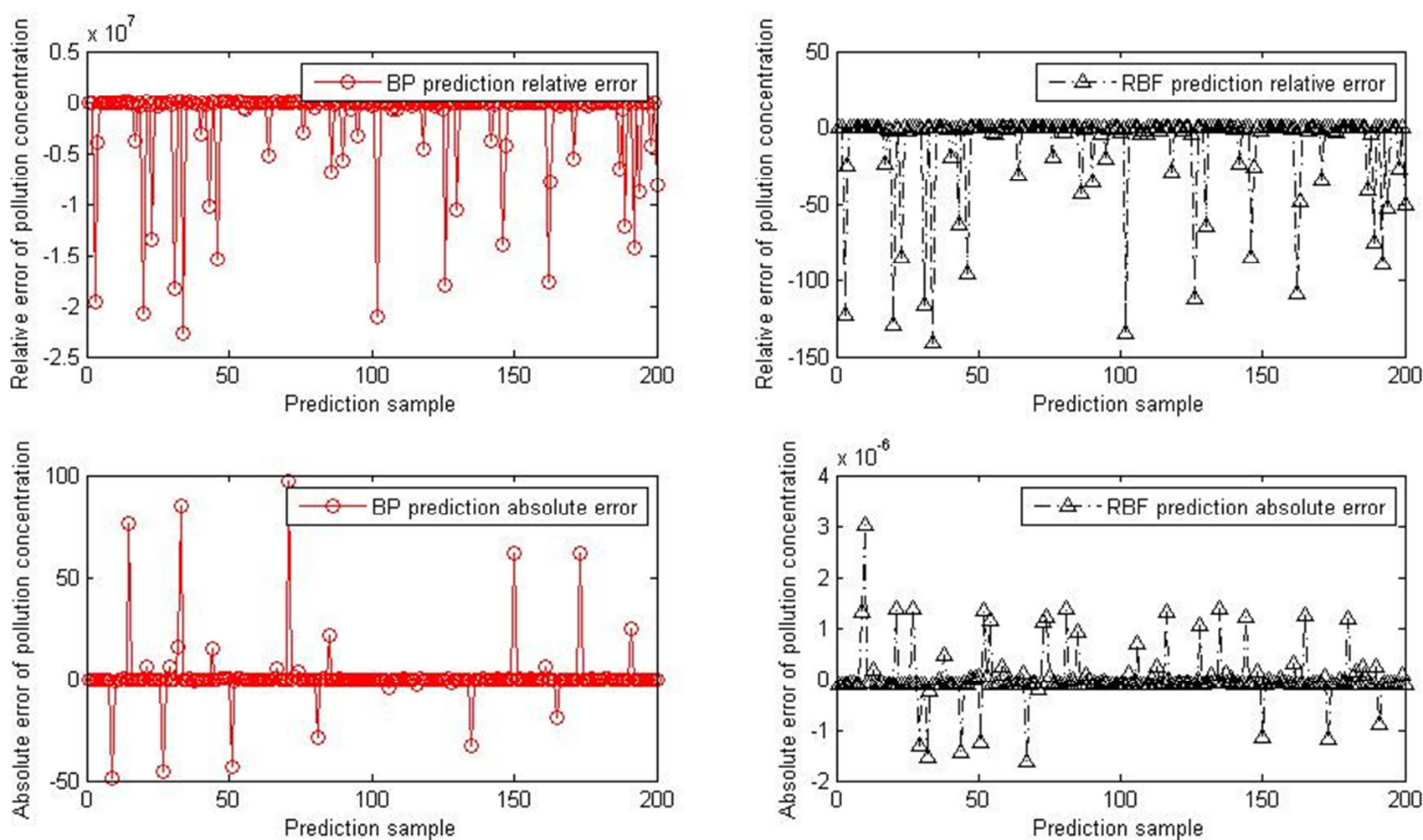

Figure 2. Absolute Error And Relative Error Of Two Algorithms.

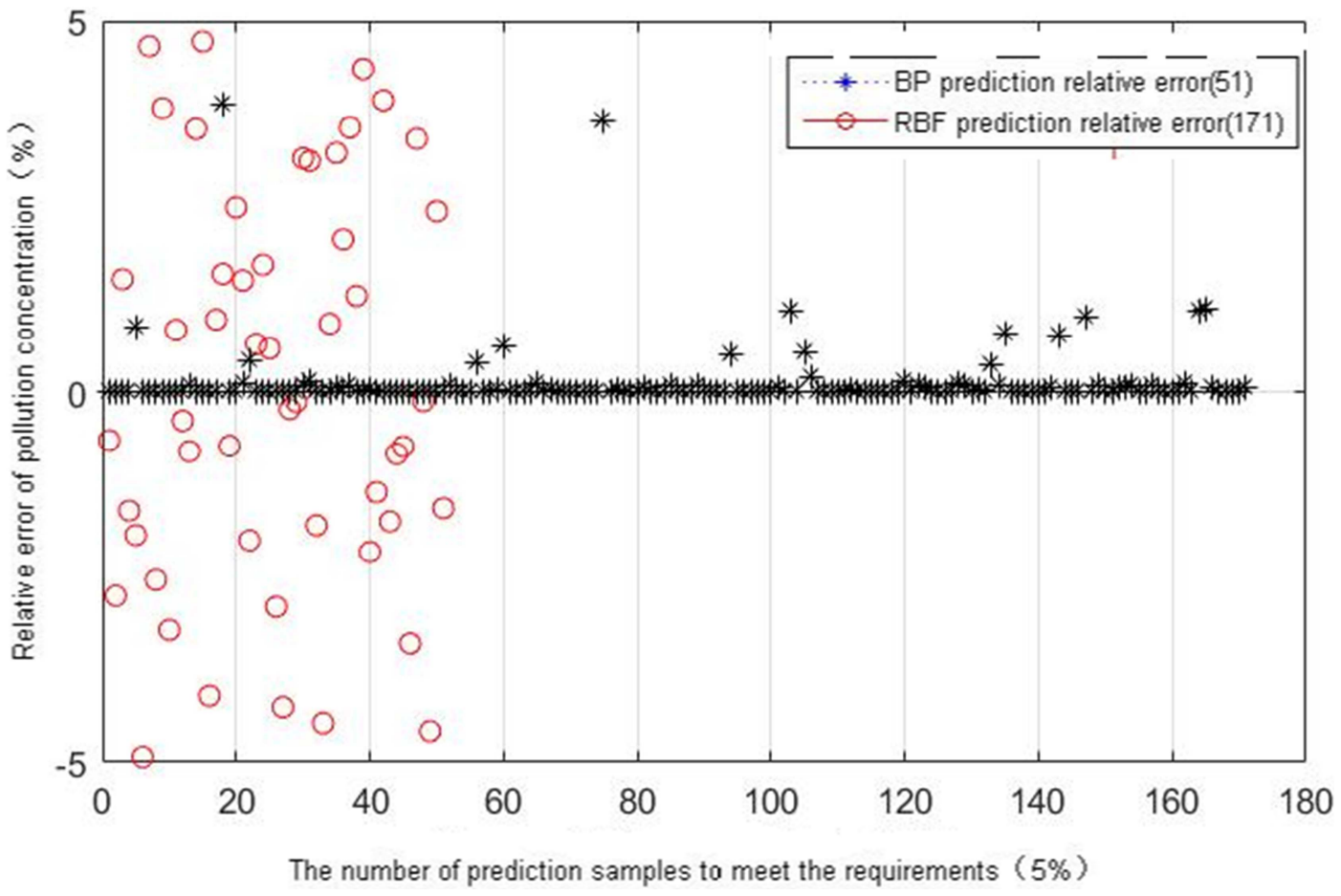

Figure 3. Number Of Prediction Data Within The Error Range Of 5\%.

It was known from Figure. $1 \sim 3$ that $\mathrm{RBF}$ neural network-based pollutant concentration prediction results had smaller absolute error and relative error than BP neural network-based pollutant concentration prediction results; according to the number of prediction data within the error range of $5 \%$, RBF neural network-based pollutant concentration prediction accuracy was much higher than BP neural network-based pollutant concentration prediction accuracy. Thus, RBF neural network-based prediction results were more accurate.

\subsection{Data Prediction-Based Sensor Array Design}

The sensor network was used for pollution monitoring. The sensors were arranged in the monitoring area in a certain array as shown in Figure 4. 


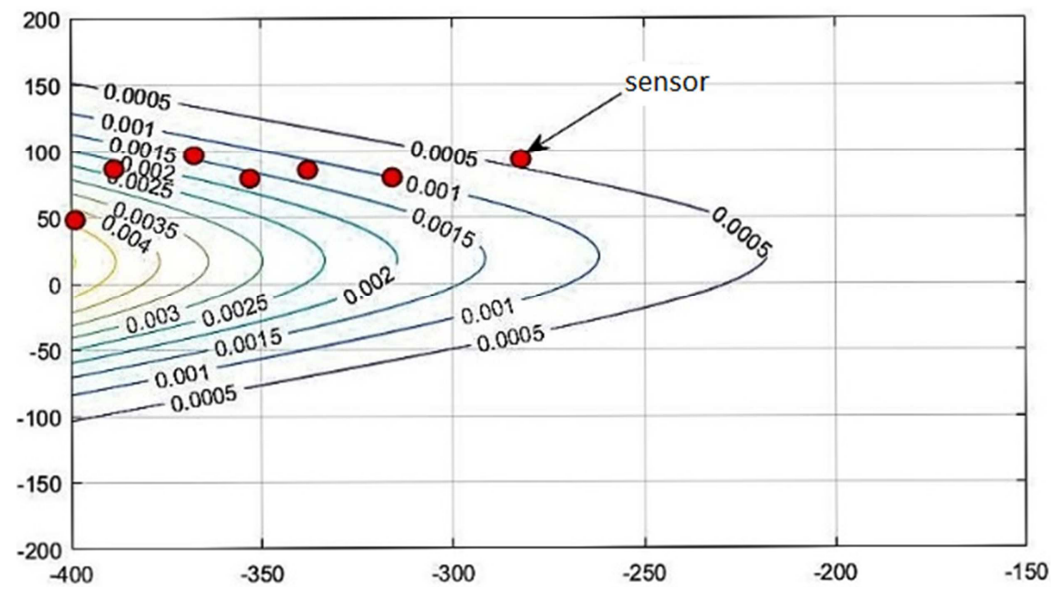

Figure 4. Air Pollution Sensor Network Arrangement.

The sensors were generally arranged according to the finite difference method as shown in Figure 5 (a). The sensors were arranged in the form of array. The arrangement of a sensor at each position in the array would lead to a waste of sensors and an increase of cost because of broad air pollution ranges. According to Chapter 1, RBF neural network -based air pollutant concentrations measured by the arranged sensors were used for estimation of the air pollutant concentrations at the unknown positions, resulting in effective reduction of observation cost. The sensor data acquisition and prediction points could be arranged in the data observation network according to Figure 5 (b).

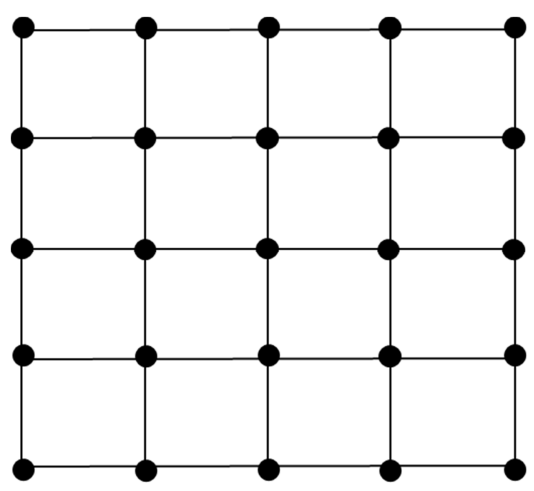

(a) Array

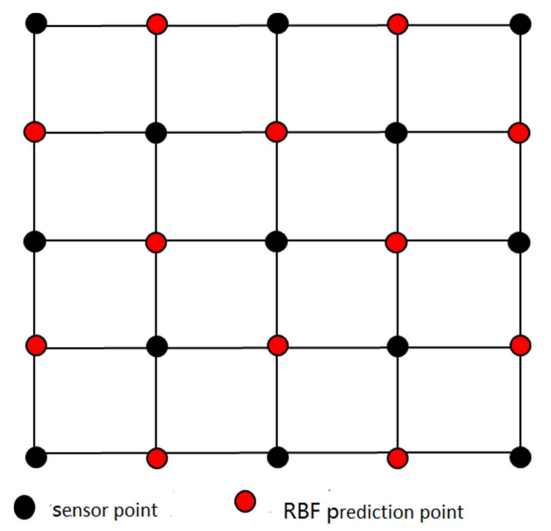

(b) Actual Sensor Arrangement

Figure 5. Sensor Array Arrangement Scheme.
To acquire and predict the pollutant concentrations more accurately, the data were acquired and processed by means of segmented sampling \& segmented prediction. The sensors were arranged more densely at the positions with higher value of concentration, and more sparsely at the positions with lower value of concentration as shown in Figure. 6.

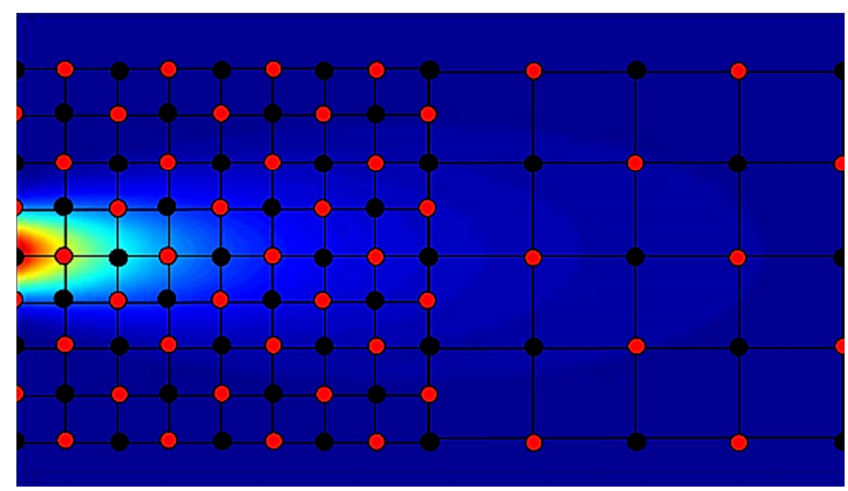

Figure 6. Air Pollution Sensor Network Arrangement.

\subsection{Inverse Calculation Model for Air Pollution Source}

\subsubsection{Gaussian Plume Diffusion Model}

The effect of landform on pollutant diffusion was taken into account in this paper. The plume diffusion was regarded as bounded, so the air pollutant diffusion could be simulated by bounded Gaussian plume diffusion model. Assuming that the coordinate of the pollution source was $\left(x_{0}, y_{0}, z_{0}\right)$, its equation was shown in Formula (3) [24]:

$$
\begin{aligned}
C(x, y, z)= & \frac{Q}{(2 \pi)^{2 / 3} \sigma_{x} \cdot \sigma_{y} \cdot \sigma_{z}} \cdot \exp \left[\frac{-\left((x-u \cdot t)-x_{0}\right)^{2}}{2 \sigma_{x}^{2}}\right] \\
& \cdot \exp \left[\frac{-\left(y-y_{0}\right)^{2}}{2 \sigma_{y}^{2}}\right] \\
& \cdot\left\{\exp \left[-\frac{\left(z-z_{0}\right)^{2}}{2 \sigma_{z}^{2}}\right]+\exp \left[-\frac{\left(z+z_{0}\right)^{2}}{2 \sigma_{z}^{2}}\right]\right\}
\end{aligned}
$$


Where

$C(x, y, z)$ : Pollutant concentration at $(x, y, z)$;

$Q$ : Pollution source intensity;

$u$ : Current downwind wind speed;

$\sigma_{x}, \sigma_{y}, \sigma_{z}:$ Air pollution diffusion coefficients.

The corrected Gaussian plume diffusion model considering the effect of ambient temperature on pollutant diffusion was shown in Formula (4):

$$
C_{m}(x, y, z)=\frac{10^{6} T \cdot C(x, y, z)}{(T+273)}
$$

Where

$C_{m}(x, y, z)$ : Corrected pollutant concentration at $(x, y, z)$;

$T$ : Current ambient temperature.

The air pollutant concentration at one point in the multi pollution source space could be approximatively regarded as the linear superposition of pollutant concentration of multi pollution source at this point. Assuming that there were $N_{c}$ different pollution sources in this space, total pollutant concentration at $(x, y, z)$ was calculated according to the formula below:

$$
C_{z}(x, y, z)=\sum_{i=1}^{N_{c}} C_{m}^{i}(x, y, z)
$$

\subsubsection{Quadratic Optimization of Pollution Source}

Plumes diffuse downwind during air pollutant diffusion, resulting in small impact on upwind environment. All the pollution areas were simulated in 3-D space downwind under the conditions. Thus, we could calculate the values for $N_{c}$ different pollution sources in the space in the following steps:

Step1: Creation of the target functions with minimal error about the concentrations and positions of finite pollution sources, i.e.:

$$
\min f(Q)=\sum_{j=1}^{N_{e}}\left(C_{m e s}^{j}(x, y, z)-C_{z}^{j}(x, y, z)\right)^{2}
$$

Intelligent optimized algorithm was used to calculate the concentrations and positions of the pollution sources according to Formula (6).

Step 2: The optimized pollution sources were ranked along $\mathrm{x}$-axis in the ascending sequence to obtain the optimized pollution source points $Q_{1}\left(x_{1}, y_{1}, z_{1}\right), Q_{2}\left(x_{2}, y_{2}, z_{2}\right) \ldots$ $Q_{i}\left(x_{i}, y_{i}, z_{i}\right) \ldots Q_{N c}\left(x_{N c}, y_{N c}, z_{N c}\right): x_{1}<x_{2}<\cdots x_{i} \cdots<x_{N c}$, $i \in\left(\begin{array}{ll}1 & N_{c}\end{array}\right)$.

The pollutant concentration at $Q_{1}\left(x_{1}, y_{1}, z_{1}\right)$ was measured to obtain $Q_{\text {mes_1 }}\left(x_{1}, y_{1}, z_{1}\right)$. If

$$
\left|\frac{Q_{m e s \_1}\left(x_{1}, y_{1}, z_{1}\right)-Q_{1}\left(x_{1}, y_{1}, z_{1}\right)}{Q_{m e s \_1}\left(x_{1}, y_{1}, z_{1}\right)}\right| \times 100 \%<5 \%,
$$

it could be believed that the first pollution source predicted in this step was accurate. The data of this pollution source was used for positive calculation to obtain the pollutant concentration contribution, $C_{1}^{j}\left(x_{1}, y_{1}, z_{1}\right)$, of this pollution source at this monitoring point $j$ to each monitoring point.

Step3: The measured pollutant concentration $C_{\text {mes }}^{j}(x, y, z)$ was deducted from $C_{1}^{j}\left(x_{1}, y_{1}, z_{1}\right)$ to obtain the total pollutant diffusion concentration which the pollution source $N_{c}-1$ contributed to.

Step4: According to Steps1 3, the pollution source $N_{c}-1$ was inversely calculated.

Among the optimized algorithm ins Step1, genetic algorithm, particle swarm optimization algorithm and Pattern Search Method and other were usually used [9, 10, 25]. This paper aims to solve the problem of nonlinear quadratic optimization in Formula (6) and minimize the error sum of square in Formula (6) in order to identify the pollution sources.

To solve the problems about single pollution source and multi pollution source, genetic algorithm, particle swarm optimization algorithm and differential evolution algorithm were used for comparative tests in order to achieve ideal inverse calculation of pollution source intensity and position under different conditions in this paper.

\section{Result and Discussion}

For single pollution source, the pollution source intensity was taken as $Q=5 \times 10^{6}$, source position as $\left(x_{0}, y_{0}\right)=(0,50)$, atmospheric stability as Grade $\mathrm{F}$, and average wind speed as $2 \mathrm{~m} / \mathrm{s}$. Figure 7 showed the identification results of pollution source position and concentration from inverse calculation of source intensity by differential evolution algorithm, adaptive particle swarm optimization algorithm and genetic algorithms. It could be seen from Figure 7 that the pollution source position and source intensity identified by adaptive particle swarm optimization algorithm were closer to the true values.

For multi pollution source, the first pollution source intensity was taken as $Q_{1}=5 \times 10^{6}$, and source position as $\left(x_{1}, y_{1}\right)=(0,50)$; the second pollution source intensity was taken as $Q_{2}=8 \times 10^{7}$, and source position as $\left(x_{2}, y_{2}\right)=(20,0)$. The results of inverse calculation of multi pollution source position and intensity under the same other conditions were showed in Figure 8. The simulation results showed that this algorithm had worst inverse calculation results of position and intensity of multi pollution source than those of single source; genetic algorithm had more accurate inverse calculation results of pollution source position and intensity than the other two algorithms. 


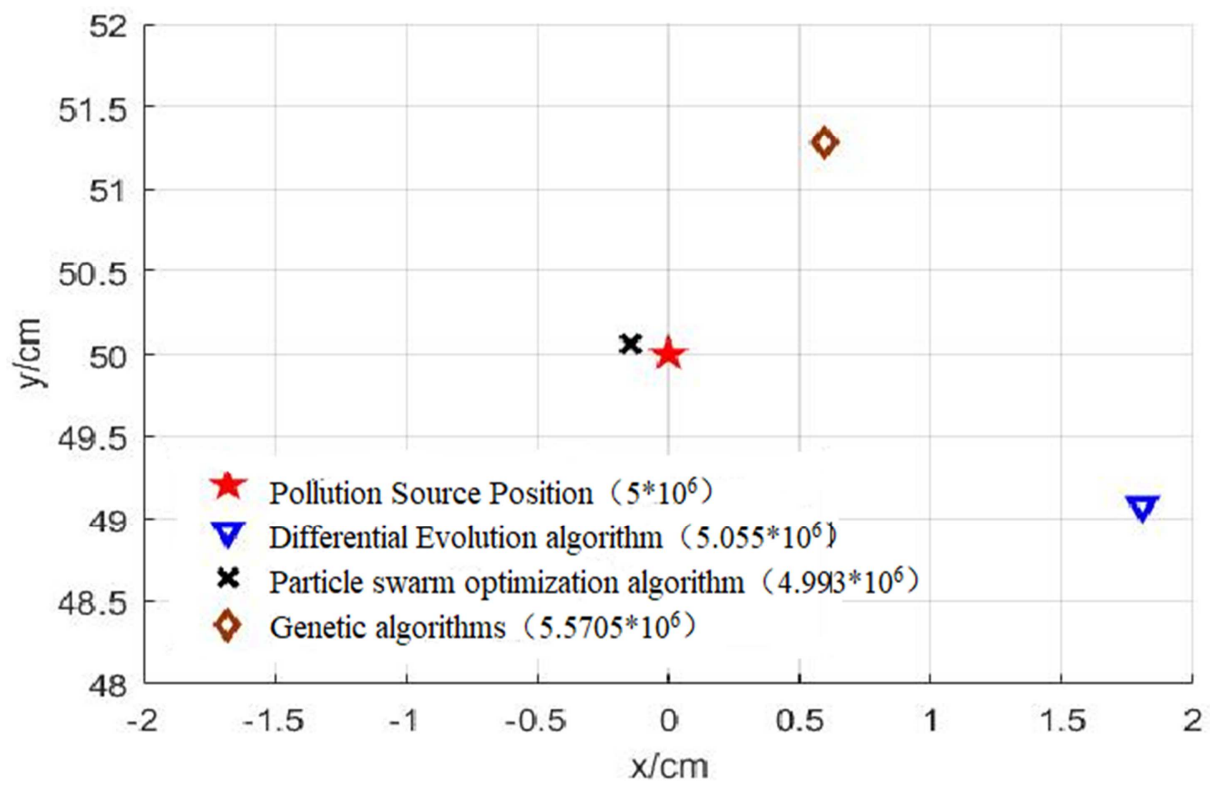

Figure 7. Single Pollution Source Position And Concentration Inversion Results.

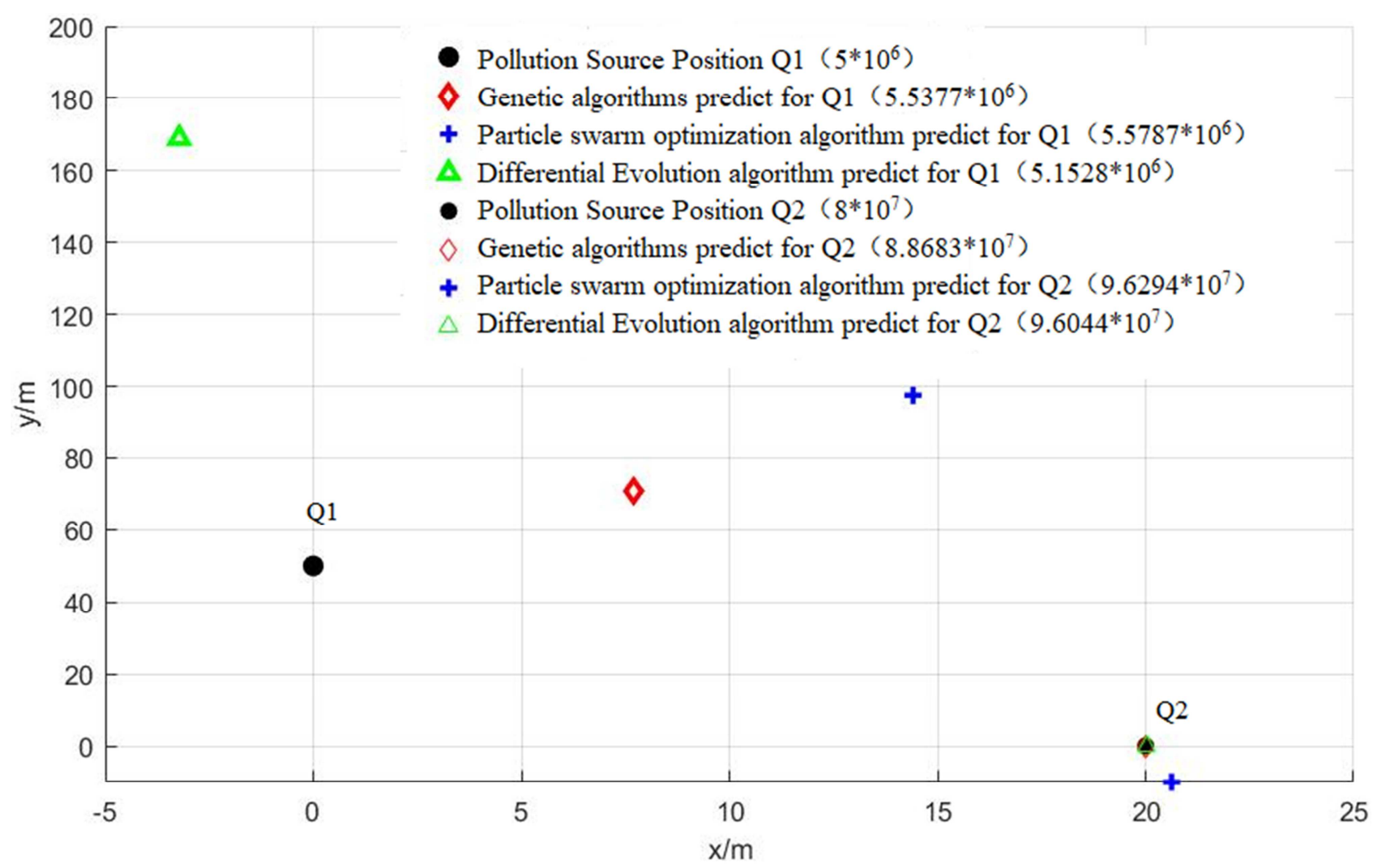

Figure 8. Double Pollution Source Position And Concentration Inversion Results.

To verify the inversed pollution source diffusion effect, the inversed pollution source diffusion residual mean was defined as follows:

$$
R=\sqrt{N_{e} \sum_{j=1}^{N_{e}}\left(C_{\text {comp }}^{j}(x, y, z)-C_{m e s}^{j}(x, y, z)\right)^{2}}
$$

Where
$C_{\text {comp }}^{j}(x, y, z)$ : The calculated air pollutant concentration at the $j$ th point,

$C_{m e s}^{j}(x, y, z):$ The measured air pollutant concentration at the $j$ th point,

$N_{e}$ : Number of sensors installed downwind of the air pollution source.

The error analysis was shown in Table 1. 
Table 1. Air Pollutant Emission Inversion Residual Mean

\begin{tabular}{lll}
\hline Item & Optimized Algorithm & Residual Mean \\
\hline \multirow{2}{*}{ Single pollution source } & Differential Evolution algorithm & $1.0306 \times 10^{-9}$ \\
& Particle swarm optimization algorithm & $8.0457 \times 10^{-10}$ \\
& Genetic algorithms & $9.4853 \times 10^{-10}$ \\
Double pollution source & Differential Evolution algorithm & $3.6094 \times 10^{-5}$ \\
& Particle swarm optimization algorithm & $2.6657 \times 10^{-5}$ \\
& Genetic algorithms & $2.0760 \times 10^{-5}$ \\
\hline
\end{tabular}

It was known from Table 1 that for single pollution source, the particle swarm optimization algorithm provided the smallest residual mean and the best inversion effect; for double pollution source, genetic algorithm provided the best inversion effect, and much higher residual mean than that for single pollution source, indicating that this algorithm was more difficult for inversion of multi pollution source, and the optimized algorithm for inversion would be selected based on different pollution sources.

\section{Conclusion}

Based on the pollutant data acquired by the sensors in segmented array, RBF neural network and different algorithms were used to study the air pollution diffusion inversion, and mainly simulate the intensity and position of single pollution source and multi pollution source in this paper. The followings were concluded:

(1) The pollutant concentrations predicted by RBF neural network had smaller absolute error and relative error than those by BP neural network algorithm; the RBF neural network model could be used to study the air pollution diffusion.

(2) The comparison of three different intelligent optimized algorithm showed that the pollution source position and source intensity identified by adaptive Particle swarm optimization algorithm were closer to true values.

(3) The results of simulation by the quadratic optimization model for pollution source concentration and position created by the measured data and calculated data according to the built Gaussian plume diffusion model showed that for single pollution source, particle swarm optimization algorithm provided the smallest residual mean and the best inversion effect; for double pollution source, genetic algorithms provide better inversion effect than other algorithm, but different algorithms had worse inversion effect on double pollution source than single pollution source.

In this paper, the study on single pollution source and multi pollution source by neural network model and different intelligent algorithms showed that the neural network model could be used for determination of multi pollution source position and intensity. It is very significant to determine pollution source under actual urban conditions. However, the air diffusion factors are very complicated. The determination of pollutant source intensity and position at other factors will still be further studied.

\section{References}

[1] Turner D B. Atmospheric dispersion modeling. a critical review [J]. J. Air Pollut. Control Assoc. (United States), 1979, 29 (5).
[2] Lushi E, Stockie J M. An inverse Gaussian plume approach for estimating atmospheric pollutant emissions from multiple point sources [J]. Atmospheric Environment, 2010, 44 (8): 1097-1107.

[3] Wu Zengmao, Sheng Lifang, Liu Feng. Research progress of numerical prediction methods for urban regional atmospheric quality [J]. Meteorological Technology, (In China) 1999, 1:11-15.

[4] Liley J B. Analytic solution of a one-dimensional equation for aerosol and gas dispersion in the stratosphere [J]. Journal of the atmospheric sciences, 1995, 52 (18): 3283-3288.

[5] Lin J S, Hildemann L M. A generalized mathematical scheme to analytically solve the atmospheric diffusion equation with dry deposition [J]. Atmospheric Environment, 1997, 31 (1): 59-71.

[6] Annunzio A J, Young G S, Haupt S E. A Multi-Entity Field Approximation to determine the source location of multiple atmospheric contaminant releases [J]. Atmospheric environment, 2012, 62: 593-604.

[7] Senocak I, Hengartner N W, Short M B, et al. Stochastic event reconstruction of atmospheric contaminant dispersion using Bayesian inference $[\mathrm{J}]$. Atmospheric Environment, 2008, 42 (33): 7718-7727.

[8] Annunzio A J, Young G S, Haupt S E. Utilizing state estimation to determine the source location for a contaminant [J]. Atmospheric environment, 2012, 46: 580-589.

[9] Chen J M, Xu D H, Zhu R. Application of Genet ic Algorithms to Point-source Inversion [J]. Meteorological, 2002, 28 (9): 12-16.

[10] Zhang J F, Jiang C, Wang Z, et al. PSO Algorithm for Back-calculation of Source Intensity [J]. China Safety Science Journal (CSSJ), 2010, 10: 024.

[11] Mulholland M, Seinfeld J H. Inverse air pollution modelling of urban-scale carbon monoxide emissions [J]. Atmospheric Environment, 1995, 29 (4): 497-516.

[12] Seibert P, Frank A. Source-receptor matrix calculation with a Lagrangian particle dispersion model in backward mode [J]. Atmospheric Chemistry and Physics, 2004, 4 (1): 51-63.

[13] Shen X Y, Bi Z H, Liu H F. Gas Concentration Retrieval Algorithm Based on Kalman Filtering Theory [J]. Opto-Electronic Engineering, 2008, 12: 015.

[14] Enting I G, Newsam G N. Inverse problems in atmospheric constituent studies: II. Sources in the free atmosphere [J]. Inverse Problems, 1990, 6 (3): 349.

[15] Goyal A, Small M J, von Stackelberg K, et al. Estimation of fugitive lead emission rates from secondary lead facilities using hierarchical Bayesian models [J]. Environmental science \& technology, 2005, 39 (13): 4929-4937.

[16] Johannesson G, Hanley B, Nitao J. Dynamic bayesian models via monte carlo-an introduction with examples [J]. Lawrence Livermore National Laboratory, UCRL-TR-207173, 2004. 
[17] Senocak I. Application of a Bayesian inference method to reconstruct short-range atmospheric dispersion events [C]//AIP Conference Proceedings. 2010.

[18] Feng F Wang Z F, Tang X. 2016. Development of an adaptive algorithm based on the shooting method and its application in the problem of estimating air pollutant emissions [J]. Chinese Journal of Atmospheric Sciences (in Chinese), 40 (4): 719-729.

[19] $\mathrm{Xu} \mathrm{B} \mathrm{L,} \mathrm{L} \mathrm{H,} \mathrm{H} \mathrm{Y,} \mathrm{et} \mathrm{al.} \mathrm{Design} \mathrm{and} \mathrm{Implement} \mathrm{a} \mathrm{Parallel}$ Algorithm of Gauss Plume Model for Air Pollution Dispersion [J]. Transactions of Beijing Institute of Technology, 2014, 34 (11): 1145-1149.

[20] Ferragut L, Asensio M I, Cascón J M, et al. An efficient algorithm for solving a multi-layer convection-diffusion problem applied to air pollution problems [J]. Advances in Engineering Software, 2013, 65: 191-199.
[21] Liu J. Radial Basis Function (RBF) neural network control for mechanical systems: design, analysis and Matlab simulation [M]. Springer Science \& Business Media, 2013.

[22] Hartman E J, Keeler J D, Kowalski J M. Layered neural networks with Gaussian hidden units as universal approximations [J]. Neural computation, 1990, 2 (2): 210-215.

[23] Park J, Sandberg I W. Universal approximation using radial-basis-function networks [J]. Neural computation, 1991, 3 (2): 246-257.

[24] Jiang W M, Cao W J, Jiang R B. Air pollution meteorology tutorial [M]. Meteorological Press., 1993: 77-86.

[25] Zheng X P, Chen Z Q. Back Calculation of Source Strength and Location of Toxic Gases Releasing Based on Pattern Search Method [J]. Zhongguo Anquan Kexue Xuebao, 2010, 20 (5): 29-34. 